\title{
Electrical detection of degradation in specimens of HVDC cable insulation
}

\author{
D. Jutsell Nilsson ${ }^{1}$, S. Gubanski ${ }^{1}$ \\ ${ }^{1}$ Department of Electrical Engineering, Chalmers University of Technology, Gothenburg, Sweden
}

\begin{abstract}
One of the challenges in laboratory investigation of degradation and ageing of HVDC cable insulation is related to securing, or in other words, imitating the real service environment of the material specimens. So far, the published data refer to experiments conducted in thermo-oxidative conditions, which is not the case during normal cable operation. In reality the cable insulation is protected by a metallic barrier that blocks the transfer of any substances in and out of the construction. By-products from the cross-linking reactions cannot diffuse out and any foreign substances, such as oxygen and water, are blocked from entering into the insulation. Thus, in order to generate results that are valid, these conditions must be replicated in laboratory experiments.

This contribution presents a methodology elaborated for performing ageing experiments in a hermetically sealed environment. Degradation of the material is evaluated through measurements of changes in the electrical tree inception voltage and test object capacitance over time. Securing the environmental isolation is primarily accomplished with an isolation system consisting of a glass enclosure with attached metallic electrodes. Indium is used to create a glass-to-metal seal between the glass and the electrodes. The electrode geometry is of needle-plane type and the needle injection process is semiautomated to secure a large degree of repeatability in specimen preparation.
\end{abstract}

\section{Introduction}

Understanding the effects on HVDC cable insulation caused by long-term exposure to in-service stresses is key to evaluating current and emerging materials. Experiments which yield such results can be carried out in a number of different ways, for example, by conducting full scale experiments using actual or model cables, by using cables that have already been in-service or by carrying out laboratory experiments on miniature specimens with artificial defects. Full scale experiments, and to a certain extent, model cables, are costly endeavours. Miniature specimens, of a size such that they fit in the palm of a hand, taken from an already produced cable can carry significant advantages. The voltage required for experimentation on this scale is typically in the lower range of tens of kilovolts, as opposed to the hundreds of kilovolts that is required for full scale tests. Since the voltage required is much less, the equipment and space requirements are manageable, miniature specimens with artificial defects present a tempting economical alternative. Further, a specimen from an already produced cable ensures that there are no differences between the examined material and the insulation in-service. The downside arises when trying to relate the stress level introduced by the defect to that of the stresses found in an actual cable.

There are two weakly connected issues that complicates the effort of ageing HVDC cable insulation. Currently, extruded cross-linked polyethylene is the prevailing material used for these applications [1]. The first matter which is of importance is the possible injection and transport of charge carriers which influences the field distribution. The second relates to the metallic sheath that surrounds a typical cable in-service. The cross-linking process leaves residual by-products in the insulation which has a large effect on the electrical properties [2]. Since an actual cable is surrounded by a metallic sheath, these stay inside the insulation. The metallic sheath also serves to block any foreign matter from entering the insulation. A substantial amount of research exists showing the debilitating results in the material when oxygen is available, degradation that intensifies with increasing temperature [3-6]. In a cable with a metallic sheath, these conditions are simply not present, instead the oxygen content is found to decrease with time due to the presence of anti-oxidants [4]. In order for ageing experiments to be valid, the environmental conditions that an actual cable experiences must be replicated.

\section{Constraining the specimen}

The main property of the specimen capsule is its ability to hinder interaction between the specimen and the outside world while still maintaining electrical contact with the semiconductor and the needle. This is accomplished by using an arrangement of glass and brass. The use of glass is motivated by the fact that it is one of the few materials that is transparent, has excellent dielectric properties and that any permeation through it should be negligible for this application.

A glass-to-metal joint is utilised to seal the capsule hermetically. The seal itself is a multi-step process involving indium, a chemical element that wets and bonds against glass and that has a low melting temperature. The element is commonly used when there is a need to join non-metallic materials to other non-metallic materials or metals. The result is shown in Figure 1. The cuboid specimen is located between two brass electrodes, inside a glass tube with a square cross-section. In the middle figure the transparent blueish part indicates the polymer and the black part represents the inner semiconductor layer. 

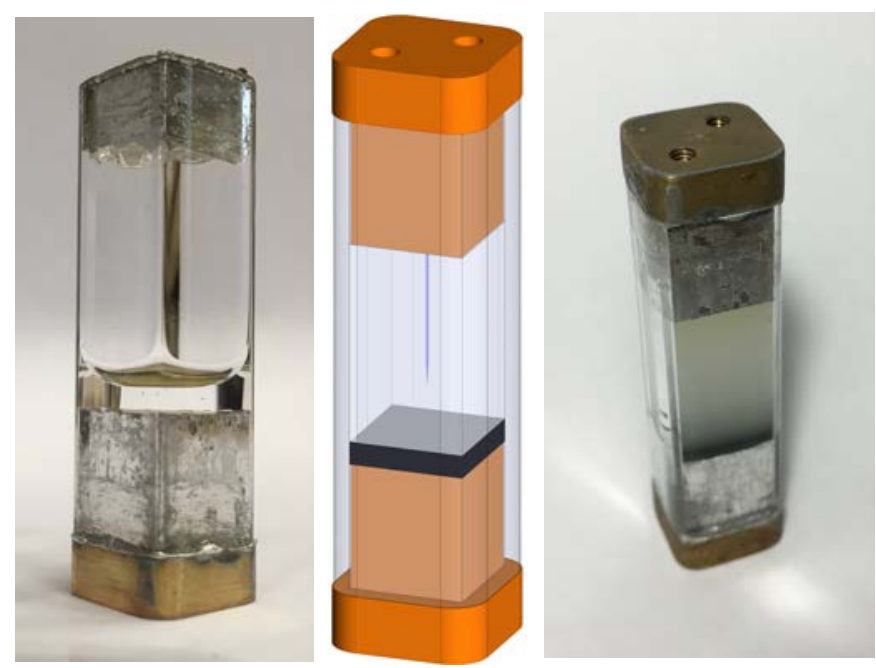

Figure 1. Left: The specimen capsule during assembly. Middle: the geometry of the specimen capsule. Right: the finished specimen capsule.

\section{Needle injection and polymer processing}

The needle injection process has substantial implications on the repeatability of the experiments. The way it is carried out influences the final geometry of the specimen, the amount of cross-linking by-products in the specimen and the polymeric composition of the final specimen. The injection process involves three steps:

- Heating the specimen to a point where the polymer is completely amorphous

- Inserting the needle while the temperature is kept stable

- Cooling the specimen back down to room temperature with the needle residing in it.

The implications for repeatability, and a part of the difficulty in this task, comes from the fact that the polymer expands when it is heated and contracts when cooled. Furthermore, the final volume of the polymer is determined by the rate of cooling. The longer time it is given to cool, the more time the specimen has to form crystalline regions. These have a higher density than the amorphous ones and thus, takes up less volume. This implies that to ensure that different specimens have the same degree of crystallinity, the temperature curve that the specimens are exposed to during injection must be almost identical, with an emphasis on the rate of cooling. The rate of loss of the crosslinking by-products from the specimen also increases with temperature, implying a need for a closed environment when the specimen is heated. An attempt to create a setup that accommodates for the above requirements is shown in Figure 2.

The specimen is surrounded on all sides by metal. The piece that sits on top of the specimen is similar to the electrode used in the capsule but made from two separate pieces in this case. Each one has a groove engraved that is just slightly larger than the needle. The two parts are then merged by soldering. The grooves line up and allows the needle passage through the whole piece. Mechanically, it is held down by four screws, keeping it in place as the specimen tries to expand. The angled orange part in the figure is a thin sheet of a copper that is used in an attempt to try and reduce the leakage from the injection cell. It is meant to deform and seal when pressure is applied. Heating elements are screwed to the sides of the specimen to facilitate heating and the temperature is regulated by a PI-controller fed with a predetermined temperature cycle. A chuck capable of centring the needle is mounted on a linear rail and controlled by a computer, allowing precise control over the needle injection.
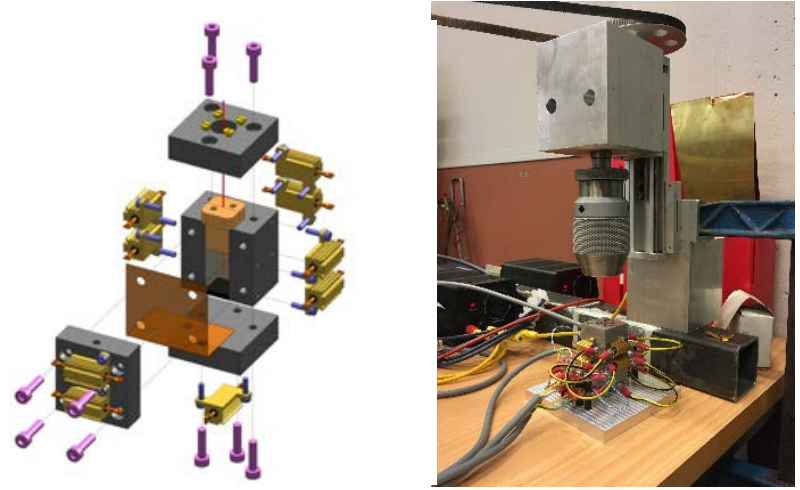

Figure 2. Left: geometry of the injection cell. Right: The finished setup.

\section{The cell}

The requirements of the cell can be summarised as follows. The specimen capsule as it is needs electric field control. Both in order to reduce the otherwise high electric fields that would develop around the sharp edges of the electrodes and in order to control the capacitance of the specimen. The measured capacitance of the electrode system should be as small as possible to better resolve small capacitive changes, it is after all the capacitive contribution from the volume in close vicinity of tip of the needle that is of interest. The specimen capsule should also be fixed in place in order to keep it from experiencing any change in its capacitive environment. The cell should support the specimen capsule axially as there is no need to stress the glassto-metal seal unnecessarily and finally, the needle tip should be optically observable. The finalised cell is shown in Figure 3.
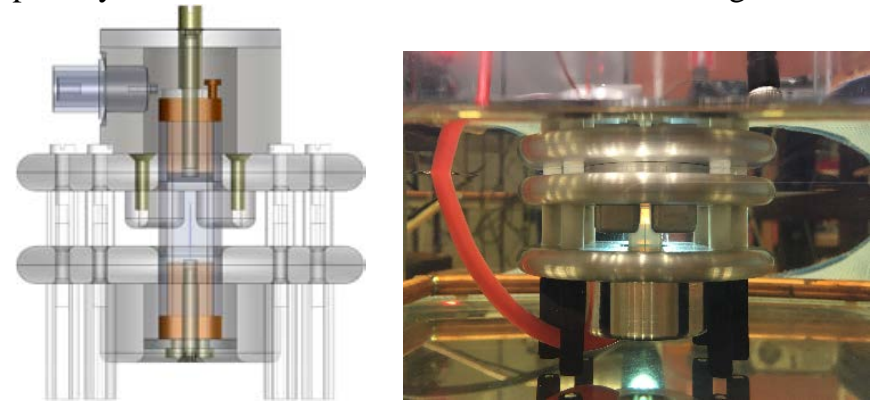

Figure 3. Left: a cross section of the cell geometry, right: the finalised cell, submerged in oil.

Ultimately, the cell is submerged in mineral oil. The oil is used both as a dielectric and as a heat transfer medium. It is necessary since the distance at the narrowest part of the cell between the two polarities is $4 \mathrm{~mm}$. This rules out keeping the cell in air during electrical tree inception experiments. The temperature in the bath is regulated using heating elements controlled by PI-controller. 


\section{Measurement setup}

Measurements that indicate the inception voltage of electrical trees were carried out using a system as shown in Figure 4. Primarily, the system consists of a reference impedance connected in series with the specimen, a voltage divider, the submerged cell as well a conventional PDmeasuring circuit: a coupling capacitor and a coupling quadripole. It is supported by computers that are responsible for supervising two temperature control loops, the recording of data from digital acquisition devices, the generation of signals using digital-to-analogue converters and exercising control of the setup in conjunction with various instruments and devices.

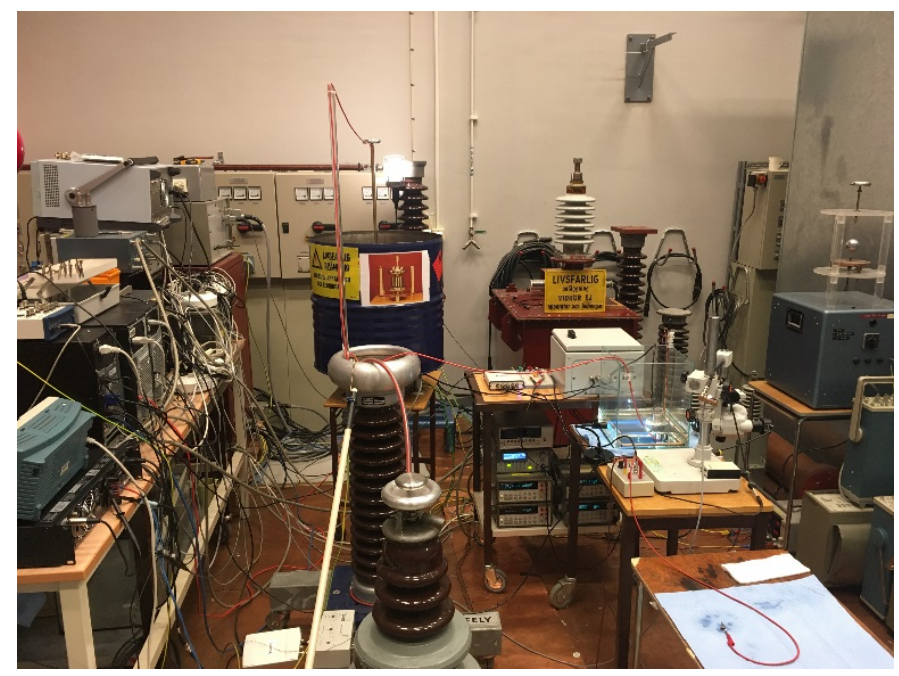

Figure 4. The setup which facilitates the electrical tree inception experiments.

As the setup is meant to be used to terminate the ageing process of a specimen by measuring the inception voltage of an electrical tree, an inherently destructive event for the specimen, the generated data point of the inception voltage is quite precious. Its value originates from the time involved ageing, the effort spent preparing the specimen, injecting the needle, sealing the capsule etc. Because of this, the data acquisition systems are configured to record a continuous stream of data from each source and save them for later analysis.

The system makes four cardinal sources of data available for analysis:

- the voltage applied to the test object

- the voltage developed over the reference impedance

- the voltage output from the quadripole

- the video stream capturing the tip of the needle, recorded by a camera through a microscope

Having access to the entire waveform produced from all data acquisition sources also enables precise analysis of the behaviour of the specimen up to and after the inception of an electrical tree, further elucidating the behaviour of the specimen examined.

The main evaluation parameter of the experiments is capacitance. Measuring it is carried out by inserting a known impedance in series with the specimen and measuring the voltage developed over it. The reference impedance consists of an oil-filled, hermetically sealed, secondary standard resistor in parallel with a capacitor constructed by embedding a metallic structure in glass. A femtoampere level operational amplifier operating in unity gain mode is used to buffer the signal. The low bias current enables measurements of high impedance specimens without skewing the results. The amplifier also isolates the reference impedance from the influence of connected data acquisition systems. The output of the amplifier acts as the input for a guard driver which feeds guard traces on the PCB. The guard continues in the screen of the cable connecting the reference impedance to the cell, either via the outer screen of a coaxial cable or the inner screen of a triaxial cable. The actively driven guard ensures that the capacitive part of the reference impedance remains constant. The passive components as well as the operational amplifiers are housed in a temperature regulated enclosure.

The excitation waveform is a ramped sinusoidal signal. The waveform is generated by a digital-to-analogue converter, amplified by a class AB-amplifier and fed to a high voltage transformer. The frequency of the applied waveform is $55.71 \mathrm{~Hz}$ and the ramp rate is $12 \mathrm{~V}_{\mathrm{rms}} / \mathrm{s}$. At the start of the measurement the various data acquisition systems are synchronised time-wise.

\section{Results}

A comparison between the detected inception voltage level across the available data sources of an experiment is shown in Table 1.

Table 1. Time and voltage of detected inception from the different data sources

\begin{tabular}{|c|c|c|}
\hline Source & Time of detection & Voltage $\left[\mathrm{kV}_{\mathrm{rms}}\right]$ \\
\hline$U_{\text {ref-imp }}$ & $17 \mathrm{~min} 37.37 \mathrm{sec}$ & \multirow{2}{*}{12.62} \\
\hline PD & $17 \mathrm{~min} 37.42 \mathrm{sec}$ & \\
\cline { 1 - 2 } Optical & $17 \mathrm{~min} 37.28 \mathrm{sec}$ & \\
\hline
\end{tabular}

Although the inception voltage can be accurately detected by any of the sources, as is evident by Table 1 , further understanding of what transpires in a specimen during an electrical treeing experiment can be gained from calculating the impedance of the specimen during the experiment.

If the specimen is modelled as an RC-parallel, the current in the circuit when the specimen is connected to the reference impedance can be expressed as

$$
i(t)=\frac{u_{1}(t)-u_{2}(t)}{R_{1}}+C_{1} \frac{\mathrm{d}\left(u_{1}(t)-u_{2}(t)\right)}{\mathrm{d} t}=\frac{u_{2}(t)}{R_{2}}+C_{2} \frac{\mathrm{d} u_{2}(t)}{\mathrm{d} t}
$$

where $u_{1}$ is the applied voltage to the specimen and $u_{2}$ is the voltage over the reference impedance, both which are measured quantities. $R_{1}$ and $C_{1}$ is the values of the model components of the specimen and $R_{2}$ and $C_{2}$ the values of the reference impedance. At this stage, the current in the circuit can be found numerically. In order to find $R_{1}$ and $C_{1}$, multiple regression is used. The method fits data that depends on more than one predictor variable. The circuit is modelled by

$A_{1} x_{1}+A_{2} x_{2}=B$ 
where $A_{1}=u_{1}(t)-u_{2}(t), x_{1}=1 / R_{1}, A_{2}=\mathrm{d}\left(u_{1}(t)-u_{2}(t)\right) / \mathrm{d} t$, $x_{2}=C_{2}$ and $B=i(t)$

Solving $\mathbf{A x}=\mathbf{B}$ for $\mathbf{x}$ is carried out by the Matlab command 'mldivide', which uses QR decomposition to find a least-squares solution of the equation system, yielding $R_{1}$ and $C_{1}$. By calculating the impedance in this way, the influence of PDactivity on the results is lessened.

Figures 5, 6 and 7 shows an example of the results of the calculations from an electrical treeing experiment. Here, the computation has been carried on a one-period basis. Figures 5 and 6 shows the same timeframe of 1.5 periods from an experiment just after that of the inception of an electrical tree. Figure 5 shows the measured current, the waveform that the calculated impedance of the specimen would have produced given the measured excitation voltage as well as the difference between the two. Figure 6 shows the measured voltage over the reference impedance as well as a numerical approximation of the expected voltage developed given the calculated impedance of the specimen. Figure 7 shows the calculated capacitance of a specimen over a timeframe that begins just before the inception of an electrical tree and during one minute of growth.

\section{Conclusions}

Overall, the goals that were set up in the onset of this work can be summarised as:

- Locate the specimen in an environment that resembles an actual HVDC-cable

- Detect the inception voltage of an electrical tree during an experiment when an increasing sinusoidal voltage is applied

The first goal can be considered to have been met. The current method of determining the success of a finalised specimen capsule is to submerge it in liquid and visually check for leakage to test the hermeticity of the seal. While this does not imply a gas tight seal, it is a substantial step in the right direction. Given the results presented, the second goal has been met successfully as every source of detection yielded an accurate value of the electrical tree inception voltage.

Out of the available detection sources, the signal from the reference impedance is arguably both the most practical and informative measure. It can be used to calculate the impedance of the specimen in which the electrical tree inception voltage is easily detected as it manifests as a distinct rise in capacitance. It also contains the base capacitance of the specimen as well as the capacitance change per time unit during tree growth. Both of the latter quantities are useful for electrical treeing experiments since they can be used as an indicator of the repeatability of the specimens and the experiments. Finally, it provides access to the current in the circuit, which is by far the best quantity to look at to get a quick overview of the outcome of an experiment.

Thus, the methodology described in this work, with an emphasis on the injection procedure and the specimen capsule, is ready for an evaluation on a larger scale. A large series, in the range of 20 specimens, would yield interesting results in terms of the repeatability of the methods developed regarding the preparation and containment of the specimen.

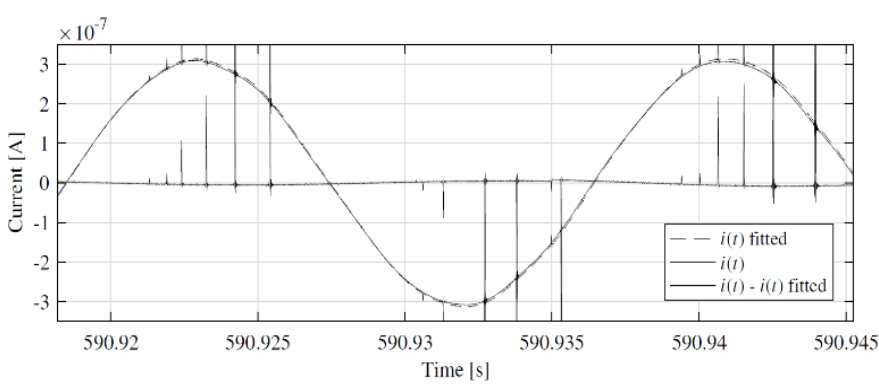

Figure 5. The current during 1.5 periods of an experiment after the inception of an electrical tree.

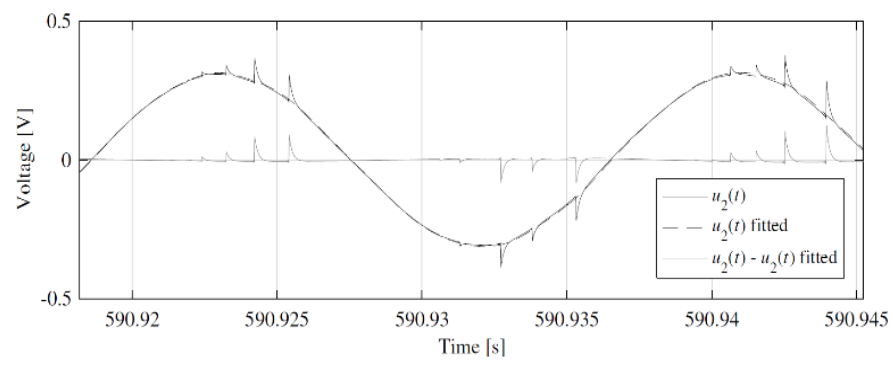

Figure 6. The voltage during 1.5 periods of an experiment after the inception of an electrical tree.

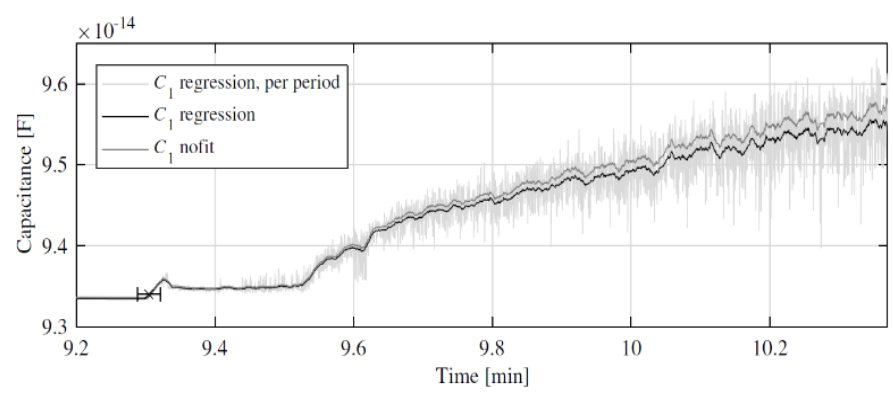

Figur 7. A window of the capacitance during electrical tree growth. The mark indicates where the electrical tree was detected optically. ' $C_{1}$ regression' and ' $C_{1}$ nofit' are results of rolling average of 43 periods, the former of the per period calculation, the latter of a $j w$ calculation.

\section{References}

[1] C. W. Reed, "An assessment of material selection for high voltage DC extruded polymer cables," in IEEE Electrical Insulation Magazine, vol. 33, no. 4, pp. 22-26, July-August 2017.

[2] T. Andrews, R. N. Hampton, A. Smedberg, D. Wald, V. Waschk, and W. Weissenberg, "The role of degassing in XLPE power cable manufacture," IEEE Electrical Insulation Magazine, vol. 22, no. 6, pp. 5-16, Nov 2006.

[3] S. M. Gubanski, K. Karlsson, and U. Gedde, "Study of thermal oxidation in medium density polyethylene,” in IEEE International Symposium on Electrical Insulation, Jun. 1992, pp. 161-164.

[4] H. Li, J. Li, Y. Ma, Q. Yan, and B. Ouyang, "The role of thermo-oxidative aging at different temperatures on the crystal structure of crosslinked polyethylene,” Journal of Materials Science: Materials in Electronics, vol. 29, no. 5, pp. 3696-3703, Mar 2018.

[5] J. Paul, E. W. Hansen, and J. Roots, "Probing the molecular dynamics in XLPE aged at different temperatures by $1 \mathrm{H}$ NMR relaxation time measurements,” Polymer Degradation and Stability, vol. 97, pp. 2403 - 2411, 2012.

[6] G. C. Montanari and A. Motori, "Thermal endurance evaluation of XLPE insulated cables,” Journal of Physics D: Applied Physics, vol. 24, no. 7, pp. 1172-1181, 1991. 